\title{
Calidad de vida en docentes universitarios: una revisión sistemática.
}

\author{
Quality of life in university teachers: a systematic review \\ relation of the triangle of sustentation \\ Soto-céspedes-Juan Carlos*4.
}

Como citar este artículo: Soto céspedes-Juan-Carlos. Relación Calidad de vida en docentes universitarios. Revistas Signos Fónicos.2018: 4(2).50-64

Correspondencia autor: jua.soto@mail.udes.edu.co (Juan Carlos Soto Céspedes)

Recibido: Junio 25 de 2018 Aprobado: Octubre 20 de 2018.

\section{RESUMEN}

INTRODUCCIÓN: la preocupación por el tema de Calidad en Docencia Universitaria ha sido más visible en entes gubernamentales y sociales que dentro de la misma comunidad docente. Por esta razón hemos iniciado este proceso de investigación con el objetivo de hallar metodologías existentes en la medición de Calidad de Vida en Docentes Universitarios a través de una extensa revisión de la literatura por medio de una revisión sistemática MÉTODOS: esta investigación corresponde a un estudio cualitativo, de corte transversal y descriptivo, que tuvo como propósito establecer cuál es el mejor método de medición de la calidad de vida en docentes universitarios utilizando metodología PICO. RESULTADOS: estos estudios revelan diferentes metodologías para la medición de la calidad de vida, entre los que se encuentran: entrevistas abiertas, entrevistas semiestructuradas, discusión de grupos, cuestionarios web, adaptación a Escala de Calidad de Vida en Adultos (EVCA), Escala GENCAT. ANÁLISIS Y DISCUSIÓN: se evidencia que el mejor método de medición de la Calidad de Vida en Docentes Universitarios, son las escalas estandarizadas, ya que, por su diseño, presentan mayor facilidad de calificar; estas se elabora solamente un formato, por lo son más rápidos, los resultados son más confiables debido al análisis estadístico. CONCLUSIONES: el concepto Calidad de Vida en relación a la docencia universitaria lo podemos ver desde distintas perspectivas, entre las que se encuentra, la calidad en el reto a la educación, en un proceso de transformación y en la forma de vida.

PALABRAS CLAVES: calidad de vida, docente universitario, escalas, entrevistas, confiabilidad.

\begin{abstract}
INTRODUCTION: Concern about the issue of Quality in University Teaching has been more visible in governmental and social entities than within the teaching community itself. For this reason we have started this research process with the aim of finding methodologies identified in the measurement of Quality of Life in University Teachers through an extensive literature review through a systematic review. METHODS: This research corresponds to a qualitative study, cross-sectional and descriptive, which aims to establish is the best method of measuring quality of life in University Teachers using PICO methodology. RESULTS: These studies reveal different methodologies for measuring quality of life, among which we find: Open interviews,

4 *Fonoaudiólogo, Maestría en Discapacidad, jua.soto@mail.udes.edu.co, Orcid: 0000-00032095-9123, Universidad de Santander -Colombia.
\end{abstract}


semi-structured interviews, group discussion, web questionnaires, adaptation to the Adult Quality of Life Scale (EVCA), GENCAT Scale. ANALYSIS AND DISCUSSION: It is evident that the best method of measuring the Quality of Life in University Teachers are the standardized scales, since, due to their design, they present greater ease of qualification; These are produced in only one format, so they are faster, the results are more reliable due to statistical analysis. CONCLUSIONS: The concept of Quality of Life in relation to university teaching can be seen from different perspectives, among which is quality in the replication of education, in a process of transformation and in the way of life.

KEYWORDS: faculty, interviews, reliability, scales, quality of life.

\section{INTRODUCCIÓN}

La "Calidad de Vida" inicia su aparición en los Estados Unidos posterior a la Segunda Guerra Mundial, en donde los intelectuales de la época deseaban comprender los puntos de vistas de las personas sobre si poseían una buena vida o si se sentían con seguridad económica (1).

De acuerdo con la Organización Mundial de la Salud (OMS), la calidad de vida se refiere a "La percepción que un individuo tiene de su lugar en la existencia, en el contexto de la cultura y del sistema de valores en los que vive, en relación con sus objetivos, expectativas, normas y preocupaciones" (2). Este se encuentra influenciado por el estado de salud física del individuo, definido por la OMS como "la descripción de la salud de la persona en un momento concreto en el tiempo, según ciertas normas identificables, habitualmente con referencia a indicadores de salud" (3), a nivel psicológico, de independencia, sus relaciones sociales, así como su relación con su entorno. De modo que, el concepto calidad de vida significa tener condiciones óptimas en relación con el entorno medido de manera objetiva, y un alto nivel de bienestar medido de manera subjetiva, que tiene en cuenta la satisfacción de necesidades a nivel individual y social (2).

Autores coinciden con este concepto, al afirmar que se puede concebir la calidad de vida como la "Combinación del bienestar objetivo y subjetivo en múltiples dominios considerados de importancia en la propia cultura y tiempo, siempre que se adhiera a los estándares universales de los derechos humanos" (4). En este sentido, la medición de la calidad de vida refleja las distintas experiencias vitales que las personas valoran, los distintos ámbitos que hacen que sea satisfactoria, y los ambientes sociales, físico y culturales que son importantes para las personas, incluyendo sus experiencias (4). De esta forma, Verdugo nos plantea la calidad de vida como "un concepto identificado con el movimiento de avance, innovación y cambio en las prácticas profesionales y en los servicios, permitiendo promover actuaciones a nivel de la persona, de la organización y del sistema social" (5).

La Carta de Ottawa elaborada por la Organización Mundial de la Salud (OMS), durante la Primera Conferencia Internacional para la Promoción de la Salud, suscrita en 1986 define la promoción de la Salud la cual tiene en cuenta propiciar los medios esenciales para optimizar la salud del pueblo y ejercer un mejor control sobre la misma. Con el fin de obtener un adecuado estado de bienestar físico, mental y social, una persona o comunidad debe tener la capacidad de identificar y efectuar sus ambiciones, de suplir sus necesidades y de adaptarse al ambiente o cambiarlo (6), (7). Se proponen dos visiones para el término bienestar: un enfoque hedonista (tradición epicúrea) y un enfoque eudaimonista (tradición aristotélica).

El bienestar hedonista hace referencia a la búsqueda de fuentes de satisfacción, placer instantáneo, y no involucra todo aquello que le produzca dolor, estrés o tensión al individuo. Por el contrario, el bienestar eudaimonista, que parte de la tradición aristotélica, mira al bienestar como un proceso continuo de mejora de las propias capacidades, encontrando satisfacción en el camino, con objetivos claros, y actuando de forma coherente y racional (8).

Aunque la Calidad de Vida en Docentes hoy es un tema obligatorio en el campo educativo y ha sido objeto de estudio de debate por algunos investigadores, no es de interés o preocupación de quienes ejercen en este campo de acción, puesto que se cree que está ligado a la labor docente, y en la disposi-

Revista Científica Signos Fónicos, 2018,4(2): 50-64. ISNN 2422-1716. 
ción de los esfuerzos de cada uno se está consiguiendo (9). La preocupación por el tema de Calidad en Docencia Universitaria ha sido más visible en las entidades gubernamentales y sociales que dentro de la misma colectividad docente. Por esta razón se realiza esta investigación, no sólo con el propósito de encontrar metodologías existentes en la medición de Calidad de Vida en Docentes Universitarios, sino también se busca lograr que el docente guie la búsqueda permanente de su Calidad de Vida. Siendo un reto primordial de las Universidades, la mejoría de la calidad de las actividades y dar respuesta idónea a las actuales exigencias de los entornos económico, social y cultural (9).

En este sentido, una universidad saludable sería aquella que incorpora la salud en sus proyectos, con el fin de mejorar la calidad de vida de quienes estudian o laboran en este lugar, influyendo de esta manera tanto en el entorno laboral como social (10).

Por otro lado, el autor Bognar (11) señala que el interés de asimilar la calidad de vida surge cuando las necesidades primordiales o primarias han permanecido insatisfechas y se alcanza a identificar el estilo de vida, definido por la OMS como "una forma de vida que se basa en patrones de comportamiento identificables, interacciones sociales y las condiciones socioeconómicas y ambientales" (12). caracterizado por las circunstancias en que se halla el individuo, las particularidades que le caracterizan y lugar donde se ubica su vivienda, el nivel escolar alcanzado, la seguridad social, la forma en que se invierte para ocio, los vínculos que se instauran con la familia, relaciones de pareja, amigos y compañeros de trabajo (10). Autores mencionan que se requiere de infraestructuras adecuadas, maquinaria, equipo en óptimas condiciones y materiales, que proporcionen la realización de las actividades de los docentes, así mismo, de áreas de recreo y descanso" (13), ligado a las relaciones humanas, que "implica que se valoren las personas, esencialmente, el trabajo participativo, el trabajo en equipo, la comunicación y la comprensión, que contribuyan a la formación de verdaderos equipos de trabajo" (13).

De acuerdo con esto, conocer las condiciones laborales con los que se cuenta en las instituciones es fundamental para que se cumplan los objetivos misionales de las entidades, además, para convivir en armonía y comodidad, ya que son los docentes, los administrativos y los no docentes, las personas que dedican su tiempo a una entidad o institución, dejando de lado parcialmente al menos ocho horas diarias a sus familias. Por ello se debe replantear el contexto laboral y permitir que los trabajadores permanezcan en plena comodidad dentro de la institución, desde el concepto de calidad de vida laboral (13).

Como podemos observar, la concepción de calidad de vida, a través de su adelanto histórico, se ha diferenciado por su permanente ampliación. Inicialmente, solo hacía referencia al cuidado de la salud personal; luego se convirtió en la inquietud por la salud particular e higiene pública, y más tarde se incluyeron los derechos humanos, con énfasis en los derechos laborales y ciudadanos. Luego, se agregó la capacidad de accesibilidad a las posesiones económicas y se extendió a la inquietud por la práctica del individuo en su vida social y su actividad cotidiana (14).

En Colombia, son pocos los estudios que se han venido desarrollando para determinar la Calidad de Vida de los Docentes Universitarios, además de las acciones en beneficio para esta. Ya que la preocupación por esta área está relacionada con la problemática de las condiciones de trabajo y situaciones medio ambientales en donde se desenvuelven laboralmente los docentes, se ve la necesidad de realizar una revisión amplia de la bibliografía, enfocada en la recolección de evidencia necesaria de los métodos que existen y se emplean en la medición de la Calidad de Vida en Docentes Universitarios, dando una mirada a los factores positivos y negativos de cada método, así como los criterios en común.

De esta manera la investigación pretende dar respuesta al siguiente interrogante: ¿Cuál es el mejor método de medición de la calidad de vida en Docentes Universitarios?

\section{MÉTODOS}

Esta investigación corresponde a un estudio cualitativo (15) de corte transversal y descriptivo (16) que tiene como propósito establecer cuál es el mejor método de medición de la Calidad de Vida en Docentes Universitarios.

Con el objeto de identificar estudios relevantes de interés, se realizó una revisión sistemática con metodología PICO. El modelo PICO es ampliamente utilizado y enseñado en la atención médica basada en la evidencia como una estrategia para formular preguntas, estrategia de búsqueda y para caracterizar estudios clínicos. PICO significa 4 posibles componentes diferentes de una pregunta clínica (15) 
(P) La población a la que va dirigida (Pacientes), especificando grupos de edad, sexo, estadio de la enfermedad, comorbilidad, nivel de riesgo, contexto, etc.

(I) La intervención que se va a evaluar (Intervención), que incluye dispositivo, procedimiento, prueba diagnóstica, exposición, factor pronóstico, factor de riesgo, agente etiológico, etc.

(C) La intervención con la que se quiere comparar.

(O)Las variables de resultado o desenlaces de interés (Outcomes), considerando los clínicamente relevantes e importantes para el paciente en el caso de estudios sobre eficacia, pronóstico o etiología; y en el caso de los estudios de pruebas diagnósticas, estimadores de rendimiento o validez diagnóstica (sensibilidad, especificidad, coeficientes de probabilidad y valores predictivos).

Este modelo pertenece a Cochrane, el cuál reúne la mejor evidencia de la investigación científica para ayudar a tomar decisiones informadas en la atención médica (15).

Para esto, se tuvo en cuenta las siguientes etapas:

\section{Formulación de pregunta en formato PICO}

A continuación se presenta en la Tabla 1 la descripción de la pregunta PICO.

TABLA 1. Formulación de pregunta en formato PICO.

\begin{tabular}{|c|c|c|c|}
\hline $\begin{array}{c}\text { P } \\
\text { (Patiente, } \\
\text { Population } \\
\text { or problem) }\end{array}$ & I (Intervention) & C (Comparison) & O (Outcomes) \\
\hline $\begin{array}{l}\text { ¿Cuál es el me- } \\
\text { jor método de } \\
\text { medición de la } \\
\text { Calidad de Vida en } \\
\text { esta poblacion? }\end{array}$ & $\begin{array}{c}\text { ¿Cuál es el método } \\
\text { considerado para } \\
\text { medir la calidad } \\
\text { de vida en esta } \\
\text { población? }\end{array}$ & & $\begin{array}{l}\text { ¿Cuales son } \\
\text { los resultados } \\
\text { relevantes? }\end{array}$ \\
\hline Faculty & $\begin{array}{l}\text { Measurement of } \\
\text { quality of life }\end{array}$ & & ¿? \\
\hline
\end{tabular}

Fuente: Autores.

Debido a que no se realizará ninguna comparación entre pruebas, se omitió este componente $(C=$ Comparison) en el diseño de la formulación de la pregunta PICO.

\section{Definición de los DeCs y los MeSH:}

El estudio tuvo en cuenta los siguientes descriptores para la revisión (Tabla 2):

TABLA 2. Descriptores utilizados en la revisión sistemática.

\begin{tabular}{cc}
\hline Descriptores (DeCS) & MeSH \\
\hline Faculty & Quality of life in university teachers \\
Quality of Life & Quality of life \\
Evaluation Studies as Topic & Evaluation methods \\
Social Welfare & University Teachers \\
Batteries & Batteries \\
Interview & Scales \\
\hline
\end{tabular}

Fuente: Autores. 
No MeSH: Evaluation methods, University professors, Quality of work life

\section{Proceso de Búsqueda:}

Los bancos de datos en los que se llevó a cabo la revisión fueron: PUBMED, TRIP PICO PUBMED, y COCHRANE LIBRARY.

\section{Proceso de filtrado:}

Elprocesodefiltradosellevóacaboteniendoencuentalossiguientescriteriosdeinclusión(Veranexo01):

- Tipos de estudio: Revisiones sistemáticas, estudios aleatorizados, ensayos clínicos, estudios de caso.

- Ventana de Tiempo: Últimos 10 años

- Especie: Humanos

- Temática: Evaluación de la Calidad de Vida de los Docentes Universitarios mediante escalas, baterías y encuestas sin importar que estos estuvieran validados o no

- Idioma: Publicados en cualquier idioma

- Tiempo de búsqueda: Desde el 10 de septiembre hasta el 28 de octubre del año 2018.

- Tipo de estudio: Estudios cuantitativos de corte transversal

- Población: Docentes universitarios sin importar su rango de edad.

Así mismo, se tomaron como apoyo para la teoría, artículos encontrados en la base de datos Scielo teniendo en cuenta los siguientes criterios:

- Ventana de Tiempo: Últimos 10 años

- Especie: Humanos

- Temática: Calidad de vida, Calidad de Vida en Docentes Universitarios

- Idioma: Publicados en cualquier idioma

- Tiempo de búsqueda: Desde el 10 de septiembre hasta el 28 de octubre del año 2018.

Los artículos elegidos para la revisión, se categorizaron en esta etapa según el nivel de evidencia y grado de recomendación propuestos por la Centre for Evidence-Based Medicine (CEBM) de Oxford (Primo, 2003), observados en la Tabla 3.

TABLA 3. Jerarquía de los estudios por el nivel de evidencia según CEBM Centre for Evidence-Based Medicine (CEBM).

\begin{tabular}{cc}
$\begin{array}{c}\text { Grados de reco- } \\
\text { mendación }\end{array}$ & Tipo de estudio \\
\hline A & Estudios de nivel 1. \\
B & Estudios de nivel 2-3 o extrapolación de estudios de nivel 1 \\
C & Estudios de nivel 4, o extrapolación de estudios de nivel 2-3. \\
D & Estudios de nivel 5, o estudios no concluyentes de cualquier nivel \\
\hline
\end{tabular}

Fuente: Centre for Evidence-Based Medicine (CEBM) de Oxford (16).

Finalmente, el grado de recomendación clínica se hizo teniendo como base el sistema GRADE a saber: A Recomendación extremadamente favorable, B Recomendación favorable, C Recomendación favorable pero no concluyente, $D$ No recomendado.

\section{RESULTADOS}

A partir de los estudios encontrados, se realizó una jerarquización del nivel de evidencia propuesta por CEBM (Centre for Evidence-Based Medicine) caracterizado por darle valor a éste tipo de estudio que abarca al problema clínico en discusión (16). Así mismo, se tuvo en cuenta el grado de recomendación según clasificación GRADE.

Los artículos seleccionados fueron en su mayoría clasificados según CEMB en estudios de revisión 
sistemática, estudios de ensayo clínico controlado, estudios de casos y controles, y ensayo clínico controlado aleatorizado, en donde predominaron los estudios de revisión sistemática en relación con la categoría GRADE corresponden a estudios de tipo B de nivel 2-3, o extraploración nivel 1, que indican una recomendación clínica favorable (Figura 1).

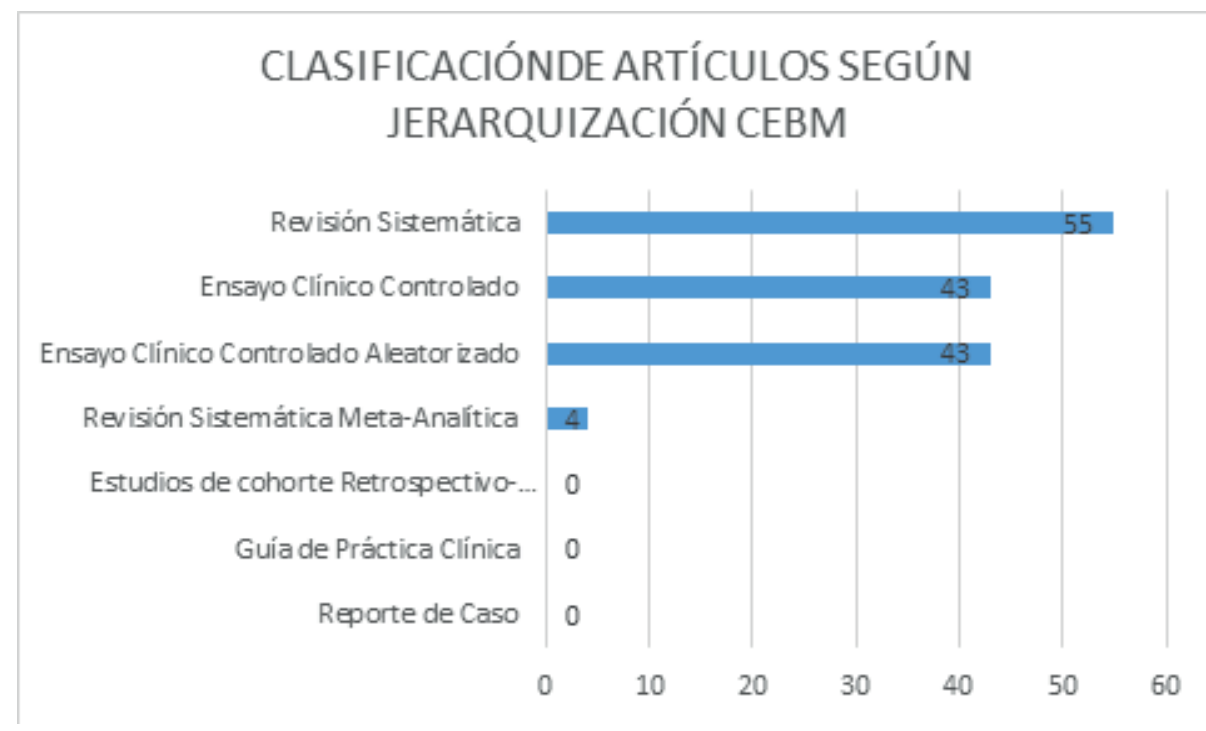

Figura 1. Jerarquización de los artículos teniendo en cuenta clasificación CEBM. Fuente: Autores.

Teniendo en cuenta los resultados en la etapa de filtrado anteriormente mencionada (Etapa 4: Proceso de filtrado), se realizó una etapa de elección de los artículos encontrados, quedando un total de 9 estudios. Los criterios de exclusión que se tuvieron en cuenta para descartar los artículos son:

- Estudios no realizados en especie humana.

- Estudios que median la calidad de vida en niños con discapacidad.

- Estiduos que no cumplian con la población estudiada.

- Estudios dirigidos a contexto clínico.

Seguidamente, los artículos que cumplían con los criterios de inclusión fueron organizados en fichas de selección de artículos que aportaba datos como (Ver anexo 03):

- Base de datos de donde se extrajo el documento

- $\quad$ nombre de el/los autor(es)

- Título

- URL

- Fecha de publicación

- Fecha de consulta

- Palabras clave

- Resumen de los artículos

Estos estudios revelan diferentes metodologías para la medición de la calidad de vida, entre los que se encuentran: Entrevistas abiertas, entrevistas semiestructuradas, discusión de grupos, cuestionarios web, adaptación a Escala de Calidad de Vida en Adultos (EVCA), Escala GENCAT, Batería de Bienestar Autorreportado (BIARE) obteniendo la siguiente evidencia:

Pruebas no estandarizadas: entrevistas abiertas, entrevistas semiestructuradas, discusión de grupos, cuestionarios web.

A partir de la década de los sesenta, científicos sociólogos desarrollan investigaciones sobre la calidad de vida recopilando información a través de encuestas, indagando el nivel de educación de las personas y estado socioeconómico, siendo considerados estos indicadores insuficientes, dado que sólo explicaban un mínimo porcentaje de la varianza en la calidad de vida individual correspondiente al $15 \%$ (17). 
Frente a esto, psicólogos realizaron propuestas de mediciones subjetivas como la felicidad, la cual la definen como el "estado de bienestar transitorio, que involucra alegría y regocijo" (18). Mientras que para otros teóricos, es un grado donde los sentimientos positivos predominan sobre los negativos (18). Para aclarar el término, sugieren que el concepto de felicidad es un estado emocional actual, diferenciándolo del de satisfacción que se da a través del tiempo, al incluir este factor y el concepto de satisfacción, se logró alcanzar un 50\% de la varianza de calidad de vida (18).

Álvarez y colaboradores en el año 2008 (9) realizaron un estudio donde se seleccionaron 3 universidades españolas: Universidad de Granada, Universidad de Valencia y Universidad Politécnica de Valencia, que tenían en común su participación en el Plan Nacional de Evaluación de la Calidad Universitaria y la Universidad Industrial de Santander de Colombia, que en ese momento estaba iniciándose en el proceso de acreditación externa, en este estudio fueron seleccionados un total de 1200 profesores. Se llevó a cabo una investigación cualitativa donde se seleccionó de acuerdo con las necesidades de información, la argumentación teórica y el estudio de casos, no solo con el objetivo de investigar para obtener documentación sino de construir a la par con los profesores implicados en el estudio (9).

Al realizar el proceso de entrevista y encuesta, se establecieron con los profesores información que no existían o que no se evidenciaba disponibles. La argumentación teórica se llevó a cabo para atraer por medio de la palabra hablada y escrita la situación de los ejercicios de los profesores en su labor docente. Y el estudio de casos se llevó a cabo para leer las concepciones en los modelos, dando valor a las ideas de los docentes entrevistados al momento de crear las significaciones de docencia universitaria y de calidad de la docencia universitaria.

La recolección de datos se llevó a cabo mediante:

- Entrevistas abiertas aplicadas a 5 profesores de cada universidad española

- Entrevistas semiestrucuradas aplicadas a 25 profesores por universidad

- Discusión de grupo, el cual permite construir un discurso sobre las categorías de cada dimensión, teniendo en cuenta la perspectiva de cada persona que lo conforma.

- Cuestionario web en donde a cada profesor de estas cuatro universidades que dispusieran de un correo institucional, se le envió un mensaje con una dirección Web para invitarlo a responder un cuestionario conformado por afirmaciones categóricas y cuatro opciones para manifestar su grado de acuerdo o desacuerdo con la afirmación

La organización de la información de llevo a cabo mediante la trascripción, codificación y categorización de grabaciones a modo de variables que forman parte de cada una de las siguientes dimensiones:

- Acción comunicativa relacionada con el reclamo de espacios propicios para realizar funciones académicas como la docencia, investigación y gestión; y sobre todo para llegar al cumplimiento de la misión de la universidad.

- Acciones pedagógicas referente a los conocimientos que debe poseer el docente para responder a lo que espera de él la sociedad.

- Profesionalidad docente orientada al aprendizaje y la formación de otros docentes

- Ambiente académico visto como el contexto en el que transcurre la vida universitaria

En las entrevistas realizadas a profesores universitarios se encontró que algunos docentes consideran que la calidad siempre se puede mejorar, por ejemplo, uno de los profesores universitarios entrevistados aporta que: "La calidad es una idea que ha entrado con mucha fuerza en todos los ámbitos de la cultura, se aplica a todo, y la usan todos pero su significado no es muy claro "(9).

La transcripción de las entrevistas abiertas y semiestructuradas permitió realizar un procedimiento de codificación que hizo asequible determinar las dimensiones y categorías sobre las cuales se originaron los conceptos de docencia universitaria y de calidad de la docencia universitaria.

En otro estudio, casi todos de los profesores de la Universidad de Alicante reflexionó que tenía una calidad de vida buena o muy buena y un grado moderado/alto de satisfacción con la vida. Señalando aspectos positivos a conservar (calidad de vida, satisfacción), las conductas a disminuir (consumo de fármacos, sedentarismo, tabaquismo), y recursos a aumentar (salud, satisfacción laboral y bienestar ligado al trabajo) (18).

Así mismo, se evaluó la calidad de vida profesional a través de la medida del estrés laboral (18) en la Universidad de Lleida, España. Los profesores evidenciaron su impresión personal de malestar, con un 
síndrome de Burnout moderado, con mayor afectación en mujeres, profesores de menor escalafón y menor antigüedad, son aquellos que tienen más dificultades con su promoción personal y sus salarios. Siendo el síndrome de Burnout, una respuesta ante el estrés crónico que produce el trabajo y está producido a las exigencias (horario, responsabilidad, condiciones). Este síndrome se identifica por los siguientes aspectos: Cansancio emocional, y baja realización personal (19). En la docencia universitaria la carencia de realización personal y el desorden en el trabajo, se asocian con la reducción del desempeño docente (19); estudios ratifican el origen laboral del síndrome de Burnout en los trabajadores (19), mientras que, en docentes de educación básica primaria los resultados favorecen a los trabajadores en los colegios privados (19).

En la Universidad de Costa Rica (19) una encuesta realizada a los docentes sobre los estilos de vida y elementos de su calidad de vida, se identificó que el $72 \%$ refieren elevadas prácticas de actividad física, el $43 \%$ se vincula a algún grupo social, político o religioso, efectúan dos visitas al año al médico general y al dentista, un $69 \%$ ingiere frutas todos los días, y un $66 \%$ consumen verduras, menos del $10 \%$ fuma cigarrillos diariamente, el $16 \%$ consume alcohol los fines de semana y casi el $20 \%$ una vez semanal.

Un estudio en la Universidad de Guadalajara, México, a 10 docentes con el fin de conocer la percepción sobre su calidad de vida. Arrojó que fue percibida como autosuficientes a nivel físico, mental y espiritual, dijeron tener buena economía para viajar, convivir con sus amistades y con toda la familia. La mitad de los entrevistados mencionaron tener una buena calidad de vida. Con el fin mejorar todos aludieron tener una actitud y pensamientos positivos, realizar ejercicios por lo menos de yoga y, o caminar media hora diaria, indicaron que una dieta saludable adecuada al problema de salud o enfermedad, realizar con frecuencia la lectura de un buen libro, aceptar apoyo familiar y de sus amistades (19).

En Venezuela (19) determina la prevalencia del estrés en la complacencia laboral de docentes en Institutos de Tecnología de Cabimas y Maracaibo, empleando la hermenéutica como estructura metodológica de recolección de datos. Como resultado se obtuvo que el distrés laboral se mantiene en un tercio, puesto que existe excesivas demandas de trabajo. Los docentes relatan sentir tensión cuando esperan un beneficio legal o contractual, y sus deseos profesionales quedan frustradas. El estrés laboral los afronta a escenarios que complican el ejercicio de sus funciones y responsabilidades.

\section{Escala de calidad de vida en adultos (ECVA)}

Otro método para la evaluación de la calidad de vida fue la Escala de Calidad de Vida en Adultos (ECVA) (20), éste estudio se realizó en la Universidad de Colima, México. La muestra del estudio fue conformada por 189 docentes: 135 hombres correspondientes a un $71.4 \%$ de la muestra y 54 mujeres que correspondían a el $28.6 \%$ total.

La categoría de esas fue de 24 a 71 años, con un promedio de 40 años. El 71.7\% eran docentes de tiempo completo y el $29.3 \%$ restante eran docentes hora cátedra o de asignatura. El rango de antigüedad en la cátedra fue de dos meses a 40 años, con trece años en promedio para docentes de tiempo completo y ocho años para docentes hora cátedra (20).

Para la obtención de datos sociodemográficos básicos en correspondencia al trabajo y entorno familiar del profesor universitario, se empleó una ficha de datos generales, que estuvo constituida de 27 preguntas (20). Para evaluar la calidad de vida se empleó la Escala de Calidad de Vida en Adultos (ECVA); la cual, tiene un índice de confiabilidad de 0.8660 , logrado mediante el coeficiente alfa de Cronbach, la cual mide la fiabilidad de una escala.

Este instrumento de medición es una escala tipo Likert que incluye 7 factores conformados por 24 ítems con 5 opciones de respuesta, que van desde "totalmente de acuerdo" hasta "totalmente en desacuerdo". La cuantía de reactivos por factor es la siguiente: satisfacción laboral, 8 ítems; satisfacción con la vivienda, 3; satisfacción familiar, 2; salud, 3; relaciones sociales y personalidad, 4; satisfacción comunitaria, 2, y relaciones vecinales, 2 ítems (20).

En los resultados conseguidos a partir de la diligencia de esta escala, se confirmó al evidenciarse que, en las puntuaciones generales de la ECVA de hombres y mujeres, los datos se congregan por arriba del promedio, es decir, entre las respuestas de "acuerdo" y "totalmente de acuerdo"; significando que tanto hombres como mujeres poseen también un alto grado de satisfacción en su calidad de vida (20). 


\section{ESCALA GENCAT}

En otro estudio, realizado en la Universidad Metropolitana de Venezuela, se utilizó una adaptación la escala GENCAT de calidad de vida. Este cuestionario corresponde a identificaciones identificables por medio de observación sobre la calidad de vida y consta de 69 ítems constituidos en ocho dimensiones (21) Bienestar Emocional (BE) el cuál hace referencia a estar tranquilo, seguro, sin agobios, no estar nervioso; Bienestar Físico (BF) el cual se refiere a tener buena salud, sentirse en buena forma física, tener hábitos de alimentación saludables; Bienestar Material (BM) el cual hace referencia a tener suficiente dinero para comprar lo que se necesita y se desea tener, tener una vivienda y lugar de trabajo adecuados; Desarrollo Personal (DP) el cual hace referencia a se refiere a la posibilidad de aprender distintas cosas, tener conocimientos y realizarse personalmente; Autodeterminación (AU) el cual hace referencia a decidir por sí mismo y tener oportunidad de elegir las cosas que quiere, cómo quiere que sea su vida, su trabajo, su tiempo libre, el lugar donde vive, las personas con las que está; Relaciones Interpersonales (RI) el cual hace referencia a relacionarse con distintas personas, tener amigos y llevarse bien con la gente (vecinos, compañeros, etc.). Inclusión Social (IS) el cual hace referencia a ir a lugares de la ciudad o del barrio donde van otras personas y participar en sus actividades como uno más y la dimensión de Derechos (DE) que hace referencia a ser considerado igual que el resto de la gente, que le traten igual, que respeten su forma de ser, opiniones, deseos, intimidad, derechos (22).

Estos docentes encuestados, eran profesores con dedicación tiempo completo, profesores con dedicación convencional o por horas, personal administrativo y estudiantes. El total de población encuestada: 231, organizados en una muestra representativa de la siguiente manera: 117 estudiantes, 30 personal administrativo , 14 profesores a tiempo convencional , 10 profesores a tiempo completo (21), (22).

El total de la muestra de profesores convencionales fue de 14, de la que 9 sujetos (64\%) correspondieron al sexo femenino y 5 (36\%) al sexo masculino. Se distribuyeron entre los grupos de edad de 26 a 35 y de más de 55. Los profesores convencionales alcanzaron una puntación estándar total de 84, un Índice de Calidad de Vida de 104 y un Percentil del índice de calidad de vida de 60 (24).

Para la muestra representativa de los profesores de tiempo completo se registraron 10 entrevistados, cuyas edades oscilaron entre los grupos de edad de 26 a 35 y más de 55 (24). Los profesores tiempo completo obtuvieron una puntación estándar total de 85, un índice de Calidad de Vida de 105 y un percentil del índice de Calidad de Vida de 62 (24).

En cuanto a la confiabilidad de este instrumento, se han realizado diferentes estudios para evaluar sus propiedades psicométricas. Algunos de los resultados obtenidos indican un buen nivel de confiabilidad, con valores de alfa de Cronbach 0.749 y 0.853 . Según el modelo de Escalas de Clasificación la confiabilidad es alta, con valores superiores a 0.95 en todas las dimensiones (24).

En la revisión realizada por Urzúa y Caqueo Urízar, los conceptos de Calidad de Vida y bienestar subjetivo concluyeron que no se encontraron definiciones únicas, por lo que proponen una clasificación de definiciones por categorías en cinco grupos:

a) Calidad de Vida = Condiciones de vida. Bajo este aspecto, la Calidad de Vida resultante de las condiciones de vida objetivamente medibles en una persona, tales como salud física, condiciones de vida, relaciones sociales, actividades funcionales u ocupación.

b) Calidad de Vida = Satisfacción con la vida. Esta categoría considera la Calidad de Vida como un sinónimo de satisfacción personal. Este tipo de definiciones también tiene sus opositores, ya que concede una relevancia al bienestar subjetivo desistiendo a las situaciones externas de vida como su contexto económico, social, cultural, político, incluyendo su propio estado de salud.

c) Calidad de Vida = Condiciones de vida + satisfacción con la vida. Este enfoque abarca de modo objetiva circunstancias de vida mediante indicadores biológicos, sociales, materiales, conductuales y psicológicos, los que complementarios a las impresiones subjetivas a cerca de cada área consigue ser el reflejo en el bienestar general.

d) Calidad de Vida = Condiciones de vida + satisfacción con la vida * Valores. La Calidad de Vida es definida como un estado de bienestar general que vislumbra descriptores objetivos y valoraciones subjetivas de bienestar físico, material, social y emocional, junto con el desarrollo personal. En esta

Revista Científica Signos Fónicos, 2018,4(2): 50-64. ISNN 2422-1716. 
categoría de términos podría adicionarse la planteada por la OMS.

e) Calidad de Vida = Condiciones de vida + satisfacción con la vida * Evaluación. El nivel de Calidad de Vida observado por una persona existiría influenciado por los procesos cognitivos relacionados a la valoración que la persona efectúa, tanto de sus contextos objetivos de vida como del grado de satisfacción con cada una de ellas (18).

Así pues, la calidad de vida compone una medida de autoreporte enfocado en la valoración que una persona hace de su alcance de bienestar y satisfacción. Esta apreciación es producto de la percepción que un individuo posee sobre su calidad de vida, por lo que se establece en un abordaje multidimensional, pues une distintas valoraciones del propio sujeto sobre las variadas aristas de su vida y de qué manera percibe bienestar o malestar referente a ellas.

\section{Batería: bienestar autorreportado (BIARE)}

La propuesta BIARE para calcular la satisfacción con la vida en profesores en la ciudad de Monterrey, Nuevo León, en una muestra de 26.409 personas, evidenció en el 2012 los siguientes resultados: el 47,3 $\%$ de los participantes afirmó estar satisfecho con su vida, el 36,1 \% manifestó sentirse moderadamente satisfecho, $11,8 \%$, poco satisfecho, y el $4,8 \%$ restante respondió estar insatisfecho. Un análisis de las situaciones civiles de los entrevistados evidenció que los solteros, los casados y quienes viven en unión libre son las personas más satisfechas y felices con su vida, mientras que los separados, los viudos y los divorciados son los menos satisfechos. En el estudio de felicidad percibida que se evaluó mediante el BIARE, se consideró la siguiente escala: 1) extremadamente insatisfecha/o, 2) muy insatisfecha/o, 3) algo insatisfecha/o, 4) ni satisfecha/o ni insatisfecha/o, 5) algo satisfecho/a, 6) muy satisfecha/o y 7) extremadamente satisfecha/o (16).

Para medir la calidad de vida se empleó una batería de pruebas constituida por 51 ítems, la cual se integra y ajusta por Arita $(2005 ; 2006)$ a partir de:

1. Escala de bienestar psicológico (Banks, Clegg, Jackson, Kemp, Stafford \& Wall, 1980), la cual contiene 12 reactivos que valoran el bienestar afectivo.

2. Escala de creencias de capacidad que identifica la capacidad que la persona cree poseer para afrontar la vida.

3. Escala de satisfacción con la vida (Diener, Emmons, Larsen \& Griffin, 1985), que valora la satisfacción global de esta. Se dispone de un solo factor, con 5 reactivos, que explican el 50,5\% de la varianza, y tiene un alfa de 0.74 .

4. Índice de bienestar personal (Cummins, Eckersley, Pallant, Vugt \& Misajon, 2003). La escala está constituida por tres factores: el primero hace referencia a la satisfacción por la localidad (alfa $=0.89$ ), el segundo por satisfacción personal (alfa $=0.82$ ) por último, satisfacción por el apoyo social (alfa $=0.74$ ).

5. Se agregaron tres ítems únicos: uno que mide lo que se denomina felicidad sentida, otro que mide satisfacción global con la vida y uno más que determina el bienestar global por la localidad.

Adicionalmente, se agregó un apartado en la entrevista para recolectar datos sociodemográficos y/o contextuales, tales como: sexo, número de enseres en el hogar, tamaño de la familia y si los padres son maestros o no.

Tánori sometió a prueba toda la batería integrada y ajustada por Arita para establecer los valores de validez por constructo y confiabilidad. Para la validez se alcanzaron indicadores aceptables, puesto que, se cumplió con el razonamiento de la medida de adecuación de muestreo (KMO), donde todos los datos obtenidos están por encima de 0.50 , valor considerado como mínimo para establecer si es provechoso someter los datos a un análisis factorial(16).

A través del análisis se determinó, que un total de 100 municipios evaluados, que el municipio con mejor nivel de felicidad percibida en México fue Apodaca, Nuevo León, con 5.70; en el lugar 100, o con menor felicidad percibida, se encontró el municipio de Campeche, Campeche, con un nivel de 4.90. En este contexto, el municipio de Monterrey, Nuevo León, se posicionó en el lugar 55, con un nivel de 5.30 en la escala de felicidad percibida (muy cercano al nivel 6: algo satisfecho) (19).

Los resultados derivados de la encuesta BIARE son esenciales porque son un referente general de la 
calidad de vida nacional y regional, aunque no manifiestan cómo el aspecto cultural o sectario de las poblaciones influye en la calidad de vida. El valor que poseen los bienes materiales (físicos u objetivos) para los individuos depende del contexto donde se desarrollan, de su posición social y ocupación (19).

\section{ANÁLISIS Y DISCUSIÓN}

En cuanto al análisis de los resultados, se evidenció un mayor número de metodología para medir la Calidad de Vida en Docentes Universitarios basada en: Entrevistas abiertas en donde el investigador lleva una serie de preguntas planificadas con anterioridad y las realiza de forma secuencial y dirigida sin poder realizar ningún tipo de comentario ni apreciaciones (25); entrevistas semiestructuradas donde el investigador realiza preguntas abiertas y el informante puede expresar su opinión; discusión de grupos el cuál es una técnica que manipula la entrevista grupal para agrupar información importante a cerca del problema de investigación, donde varias personas manifiestan simultáneamente a un cuestionario sistemático (26); adicionalmente, los cuestionarios realizados en la web, los cuales presentan como ventaja la posibilidad de acceder a un amplio número de personas con bajo coste y gran rapidez.Así mismo, se encontró en menor proporción estudios donde aplicaban escalas estandarizadas, como, por ejemplo, adaptación a la Escala de Calidad de vida en Adultos (ECVA), estudios donde se realizó adaptación a la escala GENCAT y la Batería de Bienestar Autorreportado (BAIRE).

Teniendo en cuenta lo mencionado anteriormente, se realizó una relación entre los criterios de igualdad presentes en los diferentes métodos de medición de la Calidad de Vida en Docentes Universitarios los cuales se pueden observar en la Tabla 4.

TABLA 4. Criterios de relación entre pruebas en formato PICO.

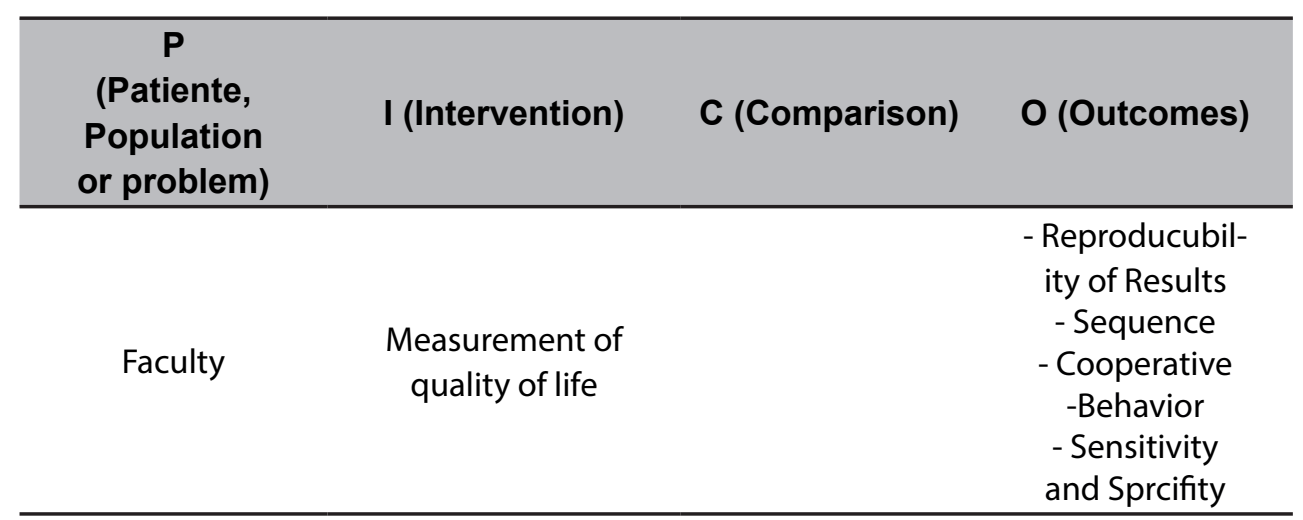

Fuente: Los Autores

La entrevista es definida por autores como "una conversación, es el arte de realizar preguntas y escuchar respuestas". Como técnica de recogida de datos, está fuertemente influenciada por las características personales del entrevistador (27). Estas, fueron las más utilizadas como método de medición de la Calidad de Vida en Docentes Universitarios y se observaron aspectos tanto positivos como negativos en su utilización. Como aspectos positivos podemos destacar que:

- En las entrevistas semiestructuradas, el investigador atañe respuestas del informante sobre una categoría, en relación a otras que se van incluyendo a medida de la entrevista y de esta forma se constituyen nuevas preguntas uniendo temas y respuestas.

- Comúnmente la encuesta presencial ha sobresalido por su superioridad en comparación con el acceso a toda la población por su alta cobertura y colaboración de los entrevistados, así como por tener sesgos de muestreo preciso y conocido.

- La experiencia al aplicar una encuesta grupal, como lo es la discusión de grupos o grupos focales, manifestó que los acercamientos en línea no sólo equiparan a los presenciales, sino que los superan, cuando cada profesor hace el esfuerzo de escribir sus ideas.

- Posibilitan en mayor proporción el contacto con toda la población objeto de estudio, considerando la capacidad que tiene cada modalidad para asegurar mejor acceso a la información 
Como aspecto negativo se logró evidenciar que:

- La transcripción de las grabaciones en relación con las entrevistas abiertas, resultaban muy extensas.

- En las entrevistas semiestructuradas el informante puede desviarse del guion inicial pensado por el investigador cuando se observan temas emergentes.

- En las encuestas abiertas, el rol del investigador involucra no sólo alcanzar respuestas, sino también comprender qué preguntas hacer y cómo hacerlas. Demanda de muchos acercamientos con los informantes y el avance es muy lento, por lo tanto, la recomendación es tratar de aprender lo que es significativo para los informantes antes de orientar los intereses de la investigación.

- En relación a los Grupo de Discusión o Grupo Focal, se centra un diálogo cuidadosamente diseñado, en donde ambiente implica ser relajado, cómodo, agradable, los participantes se inducen, responden a preguntas y contienden ideas y comentarios surgidos en la propia discusión.

Para dar respuesta a la pregunta de investigación, el mejor método de medición de la Calidad de Vida en Docentes Universitarios, son las escalas estandarizadas, ya que estas por su diseño, presentan mayor facilidad de calificar y se obtienen resultados pronto; se elabora solamente un formato, por lo tanto, es más rápido; se puede realizar una estadística sobre los resultados obtenidos siendo resultados más confiables. El abordaje de las dimensiones que estas escalas toman son los pilares que construyen las bases que concede a cada docente ser visto y verse así mismo como una persona que está en constante aprendizaje, mientras enseña y guía la formación integral del estudiante que tiene a cargo. Aun así, limita las respuestas de los informantes sin tener en cuenta los diversos puntos de vista del mismo.

Es importante a la hora de medir la Calidad de Vida del Docente, realizar un abordaje multidimensional, de manera objetiva, desde un análisis holístico, pues relaciona diversas concepciones del individuo sobre los distintos componentes de su vida y de qué manera distingue bienestar o malestar referente a ellas, puesto que la calidad de vida considera diferentes aspectos del ser humano, en el que se encuentran: físico, material, social, desarrollo y condiciones emocionales en las que se hallan, por ende, su concepto es amplio y con ello también los factores que pueden afectar la vida del ser humano.

Del mismo modo, se logró evidenciar que uno de los fenómenos estudiados acerca de la calidad de vida es el contexto laboral, este aspecto se logra cuando existe un ambiente de trabajo óptimo. La calidad de vida laboral o un ambiente laboral pleno se forma por dos aspectos, desde el ambiente físico; el cual "se refiere a que sea agradable; que las personas sientan seguridad en el trabajo; experimenten deseo y gusto de realizarlo, creando así un compromiso para la obtención de productos eficientes.

Es preciso destacar que el desarrollo que cada trabajador(a) tenga en su tarea, es lo que hace posible que la organización pueda alcanzar sus objetivos, es decir, el empleo beneficia tanto a la organización como a los empleados (al ser no sólo una fuente de ingresos, sino también satisfactor de necesidades) (19)". Cada uno de estos componentes operativos implica el diseño y realización de actividades suficientes para lograr el cometido de mejorar las condiciones de vida y aprendizaje y elevar el nivel de salud de la población considerada. Entre las actividades que se pueden realizar dentro del autocuidado se pueden citar las siguientes: fomento de la higiene, el ejercicio y el ocio saludable con aproximación al arte, sexualidad sana, prevención del consumo de drogas, alcohol y cigarrillo, prevención de la dependencia de las redes sociales etc.(20).

En la aplicación del tamiz realizado, fue posible identificar que los hábitos orales inadecuados como la respiración oral, deglución atípica, succión digital, interposición lingual y otros aspectos parafuncionales, provocan disfunciones estomatognáticas en el ser humano; puesto que son factores dañinos que generan diferencias cuantitativas con respecto a la anatomía del paladar duro y prevalencia de maloclusiones (16).

Estas maloclusiones se definen como cualquier anomalía del crecimiento óseo del maxilar o la mandíbula y/o de las posiciones dentarias que dificulten un adecuado funcionamiento del aparato masticatorio, lo que genera alteraciones sobre los propios dientes, las encías y los huesos que los soportan, la articulación temporomandibular y la estética facial (17), (18). 
Un correcto diagnóstico, es esencial para la estructuración de un adecuado plan de intervención, lo que genera a su vez, a un mejor pronóstico para el paciente frente a la alteración o maloclusión que presenta. Para esto, es fundamental efectuar un análisis cefalométrico que nos proporcione la información necesaria, con un alto porcentaje de validez y confiabilidad (19), (9).

Es importante mencionar, que el crecimiento cráneocervical se estudia generalmente en relación a la edad cronológica, sin embargo, no siempre se puede evaluar el desarrollo y la maduración somática del paciente, ya que la correlación entre la maduración ósea y la edad son fluctuantes e inseparables en cada sujeto; en otro sentido, tanto la velocidad como el ritmo de crecimiento no tienen un patrón establecido en relación a la edad (19), (20).

Diferentes autores han demostrado que el estado de maduración ósea, es determinado a través de la morfología de las vértebras cervicales (CVM) y se puede utilizar como un indicador biológico confiable (21). Es por eso que en esta investigación se tuvo como punto de referencia y hace parte del triángulo craneocervical la relación del lóbulo occipital y la primera vertebral cervical (atlas).

Uno de los estudios preliminares más importantes para realizar un correcto plan de tratamiento, son los análisis cefalométricos radiológicos, como lo recomienda (22), además considera que la atención de los odontólogos debe ir más allá de los dientes, incluyendo estructuras como los maxilares, la cabeza y el cuello. De esta forma abarca al paciente en su totalidad, teniendo en cuenta las consideraciones en cuanto la edad, el sexo y el crecimiento facial. Por estas razones en sus investigaciones, Ricketts ha logrado establecer un análisis cefalométrico muy preciso, el cual incluye diversos áreas como biotipo facial y clase esqueletal (23).

Es considerable e importante el aporte que hace el autor Ricketts puesto que es indispensable trabajar con panorámicas que brindan la posibilidad de identificar de una manera mayormente objetiva la dificultad que puede llegar a presentar el usuario. En este trabajo se analizaron radiografías de perfil en los diferentes menores que cumplían con los criterios de inclusión, dando como resultado relación entre algunos puntos como el alto del paladar y el ángulo PPA, al igual que el ángulo AOA y el ancho del paladar.

El índice de Pont Korkhaus ha sido implementado con el objetivo de pronosticar los aumentos en la medida de las arcadas dentarias durante el crecimiento. Además de poder predecir un futuro resultado de las intervenciones ortodóncicas y ortopédicos que conlleven expansión maxilar para así generar estabilidad en estas estructuras (24), (25). A pesar de que este índice es netamente para los odontólogos, en el ámbito de la Fonoaudiología es importante porque brinda la posibilidad de evaluar de manera objetiva la anatomía del usuario en este caso el paladar duro, por lo que posibilita realizar un diagnóstico más certero y veraz respecto a las características anatómicas de éste (26), (27), (28).

En esta investigación uno de los criterios de exclusión correspondió a usuarios que no debían tener ninguna aparatología fija de paladar o que presentaran alguna patología de base. Por otra parte, se incluyeron aquellos niños con tratamiento ortodóntico.

El tratamiento fonoaudiológico se basa en dar el tono muscular adecuado, por ejemplo al utilizar la mioterapia en la zona facial y mejorar los problemas en la postura como la xifosis a nivel cervical; la cual se produce para optimizar la respiración como el tipo y modo respiratorio del usuario que presente disfunción respiratoria cuando hay presencia de los hombros inclinados hacia adelante, y escápulas hacia atrás. Referente al problema anterior, dentro de la población objeto de estudio cuatro de los menores presentaron esta alteración acompañada de respiración oral, lo cual puede ser una característica física que pudo haber generado el hábito oral inadecuado.

Por otro lado, el ortodoncista parte de la maloclusión dental para corregir la oclusión, deglución, masticación. Este tratamiento ortodóntico implementa algunas veces la extracción en ciertas piezas dentarias permanentes e interconsultas con otros profesionales para el trabajo en conjunto y llevar un tratamiento eficaz. 


\section{CONCLUSIONES}

No existe una presunción única para la definición de calidad de vida, su estudio involucra enfoques médicos, económicos, sociales, políticos, psicológicos y humanísticos. Su análisis en la actualidad comprende los métodos de valores personales, culturales y el contexto en el que habitan las personas.

Las pruebas de mayor confiabilidad son las pruebas estandarizadas siendo estas el mejor método de medición de la Calidad de Vida en Docentes Universitarios, ya que da una mirada holística a esta, viendo al docente como un todo y no como una variable, abarcado diferentes aspectos en el que se encuentran: físico, material, social, desarrollo y las condiciones emocionales en las que se hallan, tomando un análisis tanto subjetivo como objetivo aportando valores de validez y confiabilidad. Por el contrario, las entrevistas sólo nos proporcionan un abordaje subjetivo.

Se evidencia que la metodología más usada para la medición de la Calidad de Vida en Docentes Universitarios son las entrevistas puesto que no limitan al docente en su respuesta, sino que por el contrario, permite al investigador ir relacionando los temas y respuestas del entrevistado.

Se hacen ineludibles los abordajes de la calidad de vida del docente universitario desde diversas perspectivas, intentando conjugar las interrelaciones de sus múltiples componentes y dimensiones.

Ya que la preocupación por esta área está relacionada con las condiciones de trabajo y situaciones medio ambientales en donde se desenvuelven laboralmente los docentes, es necesario priorizar acciones de promoción de calidad de vida dirigidas a la comunidad universitaria como entorno, es decir creando ambientes saludables, además de aquellas que fomentan cambios de hábitos de vida.

\section{REFERENCIAS BIBLIOGRAFICAS}

1. Urzúa A, Caqueo A. Calidad de vida: Una revisión teórica del concepto. Ter Psicol.2012;30(1):61-71. Doi.org/10.4067/S0718-48082012000100006

2. Robles I, Rubio B, Vanessa De la Rosa E, Nava H. neralidades y conceptos de calidad de vida en relación con los cuidados de salud. Medigraphic. 2016;11(3):120-5.

3. WHO. World Health Organization. The world health report 1998: life in the 21st century A vision for all. World Heal Organ. 1998;226.

4. Tamarit J. La psicología frente a la calidad de vida y la inclusión social de las personas con discapacidad intelectual o del desarrollo.Psychol Pap.2018;39(2).

5. Rey JL, Sanz LM. El empleo de las personas con discapacidad. 1st ed. Madrid:Dykinsom; 2018.

6.Fernández L, Gutiérrez M. Bienestar social, económico y ambiental para las presentes y futuras generaciones. Inf Tecnol. 2013;24(2):121-30. Doi.org/10.4067/S0718-07642013000200013

7. Cross R, Nohria N, Parker A. MITSloan Management Review No t C op y o r P os t No t C y o. Spring. 2002;43(3):83-96.

8. Gaxiola JC, Palomar J. Una mirada desde Latinoamérica. Mexico: Coedición UNISON QARTUPPI ;2016. Doi.org/10.29410/QTP.16.09

9.López R, Fortes MC, Grau C. Concepciones sobre la docencia universitaria de calidad. Docencia Univ. 2008; 9(1):31-55.

10. Becerra S. Universidades saludables: una apuesta a una formación integral del estudiante. Rev Psicol. 2013;31(2):287-314.

11.Castillo R, Rios C. Representaciones Sociales y Subjetivas de La Vida Académica de Los Estudiantes de La Universidad Nacional de San Agustín de Arequipa octubre 2013 - marzo 2015. Rumbos. 2015; 11: 96-117.

12. Guerrero, L; León A. Lifestyle and Good Health. 2010;14 (48);13-9.

Revista Científica Signos Fónicos, 2018,4(2): 50-64. ISNN 2422-1716. 
13. Roxana L, Fonseca M. Calidad de vida laboral.Revista de trabajo social.1999; 24:1148-1152.

14. Bobes J, González P, Bousoño M, Suárez E. Desarrollo histórico del concepto de calidad de vida. Monográfias Psiquiatr. 1993;5(6):5-11.

15. Mavergames C. Cochrane PICO: Using linked data technologies for evidence curation. [actualizado 2017julio; el acceso en el año 2018 abril]. Disponible en: https://eppi.ioe.ac.uk/CMS/Portals/0/FINAL-Mavergames_UCL-talk_July-2017.pdf

16.Delgado CM, Manterola C, Asenjo C, Otzen T. Jerarquización de la evidencia. Niveles de evidencia y grados de recomendación de uso actual Hierarchy of evidence. Levels of evidence and grades of recommendation from current use. Rev Chil Infectol. 2014;31(6):705-18. Doi.org/10.4067/S071610182014000600011

17. Developers IA. BPA as a Developer in Thermal Paper and Alternatives Cross section of thermal paper. 2010;1-44.

18.Urzúa A. 2 Calidad de vida: Una revisión teórica del concepto Quality of life: A theoretical review. Ter PSICOLÓgICA. 2012;30(1):61-71. Doi.org/10.4067/S0718-48082012000100006

19.Rivas HG, Montiel AD.Calidad de vida del docente universitario vista desde la complejidad. Rev Cuba Salud Publica. 2011; 37(3): 314-323.

20.Yan X, Feng S, Dai J, Wang J. Detecting epileptic electroencephalogram by Recurrence Quantification Analysis. CISP-BMEl. 2016. 2017;18:1482-6.

21.Nobuyuki H. Un estudio sobre la distribución del vómito en hospitales y centros de enfermería para prevenir la propagación de la infección por norovirus.Journal of Infectious Diseases. 2017; 91: 399-404.

22. Olivas V. Acompañamiento de personas con discapacidad en actividades programadas. Madrid: Editorial CEP; 2017.

23.Organización de las Naciones Unidas - ONU. Equidad e Inclusión Social: Superando desigualdades hacia sociedades más inclusivas.1st ed.Washington; 2006. 1-269.

24. Marina G, Ayari YA. Percepción de la comunidad universitaria sobre la calidad de vida en Venezuela: el caso Universidad Metropolitana. Ciencias Soc. 2017;5(9):132-59. Doi.org/10.18682/jcs.v0i9.711

25.Troncoso C, Amaya A. Interview: A practical guide for qualitative data collection in health research. Rev Fac Med.2017;65(2):329-32. Doi.org/10.15446/revfacmed.v65n2.60235.

26.Casas J, Repullo JR, Donado J. La encuesta como técnica de investigación. Elaboración de cuestionarios y tratamiento estadístico de los datos (I). Aten Primaria. 2003;31(8):527-38.

27. Corral Y. La entrevista en investigación cualitativa. Investig Merc. 2014;12, 34. 